This item was submitted to Loughborough's Research Repository by the author.

Items in Figshare are protected by copyright, with all rights reserved, unless otherwise indicated.

\title{
Objective evaluation criteria for stereo camera shooting quality under different shooting parameters and shooting distances
}

PLEASE CITE THE PUBLISHED VERSION

http://dx.doi.org/10.1109/JSEN.2015.2421518

PUBLISHER

(C) IEEE.

VERSION

AM (Accepted Manuscript)

LICENCE

CC BY-NC-ND 4.0

\section{REPOSITORY RECORD}

Yang, Jiachen, Yun Liu, Qinggang Meng, and Rongrong Chu. 2019. "Objective Evaluation Criteria for Stereo Camera Shooting Quality Under Different Shooting Parameters and Shooting Distances”. figshare.

https://hdl.handle.net/2134/18120. 


\title{
Objective Evaluation Criteria for Stereo Camera Shooting Quality under Different Shooting Parameters and Shooting Distances
}

\author{
Jiachen Yang, Yun Liu, Qinggang Meng, and Rongrong Chu
}

\begin{abstract}
The vigorous development of three-dimensional (3D) technology has improved the photography technology of stereo cameras constantly. However, there are no widely recognized objective evaluation criteria for stereo camera shooting quality under different shooting parameters and shooting distances. At the same time, no shooting guideline can be used for reference when people take stereoscopic images. To solve this problem, we propose the objective evaluation criteria of shooting quality of two types of stereo cameras (parallel and toed-in camera configurations) under three shooting conditions (macro shooting, short and long distance shooting). In our work, several prominent evaluation factors are built by analyzing the characteristics of each shooting condition. Based on the effective five-point scale used in our subjective experiments, the relationships between shooting factors and shooting quality are obtained and then effectively integrated together to build the overall evaluation criteria. Finally, extensive experiments have been conducted, and the results demonstrate that the proposed approach can effectively evaluate the shooting quality of stereo cameras.
\end{abstract}

Index Terms-Stereo cameras, objective evaluation, shooting principles, stereo images, parallel and toed-in camera configuration, stereo shooting.

\section{INTRODUCTION}

W ITH the development of 3D technology [1]-[4], stereo image sources are increasing around the world, which allows people to see the stereo images on the screen. However, some stereo image sources suffer from various kinds of distortions which may cause visual discomforts like dizziness, nausea, and the declining of the body balance. Normally, the obtained 3D images are thought to have ideal quality, while the improper parameter settings during the source image capturing process may cause uncomfortable stereo effect. It is important to identify the reasons of quality degradations to maintain the required quality of stereo contents. People usually attributes these visual discomforts to the lack of the stereo image processing and display technology, thus a number

This research is partially supported by the National Natural Science Foundation of China (No. 61101224, No.61271324 and No.61471260), Natural Science Foundation of Tianjin (No.12jcqnjc00500 and No.12jcybjc10400), supported by Program for New Century Excellent Talents in University (NCET12-0400), Postdoctoral Fund in China (2012M520574).

J. Yang is with School of Electronic Information Engineering, Tianjin University, Tianjin, China, and Department of Computer Science, School of Science at Loughborough University, UK. E-mail: yangjiachen@tju.edu.cn

Y. Liu is with School of Electronic Information Engineering, Tianjin University, Tianjin, China. E-mail: yunliu@tju.edu.cn

Q. Meng is with Department of Computer Science, School of Science at Loughborough University, UK.

R. Chu is with School of Electronic Information Engineering, Tianjin University, Tianjin, China. of efficient perceptual image quality assessment algorithms, which can be used to estimate the quality of the images with various kinds of distortions, have been presented [5], [6]. However, the evaluation systems still cannot assess all the visual discomforts, and the current evaluation theories mainly focus on the comparison between the stereo images before and after processing.

To address the above issues, several studies have investigated the effect of shooting conditions on the shooting quality [7], [8] which can be categorized into the subjective [9] and objective assessment methods [10]. Since subjective methods are time-consuming and impractical for online applications, objective methods have attracted more attention. A straightforward way is to study the objective perceptual shooting quality criteria by considering the factors of individual stereo cameras [11], [12]. Hasmanda et al. [13] presented a method to calculate the best setting of a pair of stereo camera based on the available camera parameters, e.g. focal length, parallax, inter-camera distance. IJsselsteijn et al. [14] presented a study to investigate the effects of manipulating inter-camera distance, convergence distance and camera focal length on perceived quality and naturalness. However, these methods only focused on studying several shooting parameters and how these parameters affected the shooting quality. Various human perceptions, such as visual fatigue, puppet-theater and cardboard effect and so on, also link to the shooting quality, many camera shooting methods have introduced human visual perception into their models [15]. Kim et al. [16] proposed a visual fatigue metric that could predict the levels of visual fatigue result from stereoscopic images by considering the impact of shooting distance and inter-camera distance. By analyzing the influence of inter-camera distance and camera focal length on cardboard effect, Yamanoue et al. [10] introduced parameter setting principles to achieve good stereoscopic image quality.

The above approaches studied the relationship between the effect of several shooting parameters or human perception and shooting quality, but so far there are still no widely recognized and effective objective evaluation criteria for stereo camera shooting quality. Inspired by the previous studies, we take advantage of individual characteristics of three shooting conditions, and propose the effective evaluation factors. By using the five-point evaluation scale in our subjective experiments, the individual mapping between subjective evaluation and each evaluation factor value is studied first and then the final evaluation criteria by considering the importance of each component are proposed. The main contributions of our 
TABLE I

Meaning of Stereo Camera Parameters

\begin{tabular}{cc}
\hline Camera parameters (physical value) & Semantic meaning \\
\hline$h(/ \mathrm{mm})$ & the shooting distance \\
$d(/ \mathrm{mm})$ & the inter-camera distance \\
\hline$f(/ \mathrm{mm})$ & the camera focal length \\
$p\left(/{ }^{\circ}\right)$ & the viewing angle \\
\hline$m(/ \mathrm{mm})$ & the foreground parallax \\
$n(/ \mathrm{mm})$ & the background parallax \\
\hline$l(/ \mathrm{mm})$ & the scene depth \\
$W(/ \mathrm{mm})$ & the CCD size \\
\hline$L_{\min }$ & the distance between the closest \\
$(/ \mathrm{mm})$ & scene and the camera center \\
\hline$L_{\max }$ & the distance between the farthest \\
$(/ \mathrm{mm})$ & scene and the camera center \\
\hline
\end{tabular}

paper are summarized as follows: (1) By analyzing the related shooting characteristics of two types of stereo cameras, we propose the five-point scale evaluation factors under different shooting distances based on the subjective experiments, and then linearly integrate them together to build the final overall objective shooting quality evaluation criteria of stereo camera. (2) Based on the relationships between shooting quality and stereo camera parameters, extensive subjective experiments have been conducted and the results demonstrate that the proposed criteria can achieve a good consistency with subjective assessment value.

The rest of the paper is organized as follows: Section II introduces the basic shooting principles; Section III describes the proposed objective shooting quality evaluation criteria of stereo camera; Section IV proposes the establishment of shooting principles and evaluations theories; Section $\mathrm{V}$ presents the experimental results and analysis; and Section VI concludes the paper.

\section{BASIC Shooting Principles}

Stereo shooting is generally divided into two types: parallel and toed-in camera configurations [15], [17]. Hoffman et al., through their experimental analysis of the parallel and toedin camera configurations, discovered that the short and long shooting distances (the shooting distance is measured from the stereo camera setup to the photographed objects) have different influence on the quality of images captured by stereo cameras [18]. When people watch natural scenes with different shooting distances, the region they are interested in is different. So the information acquired by human eyes is also different [15], [19]. For example, in macro shooting, the attention is focus on the object closest to the cameras, while in short and long distance shooting, the foreground and background as well as the regions of interest should all be considered (in this paper, the foreground refers to the scene in front of the observer's interest regions and the background refers to the scene behind the observer's interest regions). This paper aims to establish the evaluation criteria for parallel and toed-in camera configurations under three different shooting distances: macro, short and long distances. The parameters adopted to establish the objective evaluation criteria are shown in Table I.

The basic shooting principles include the following:

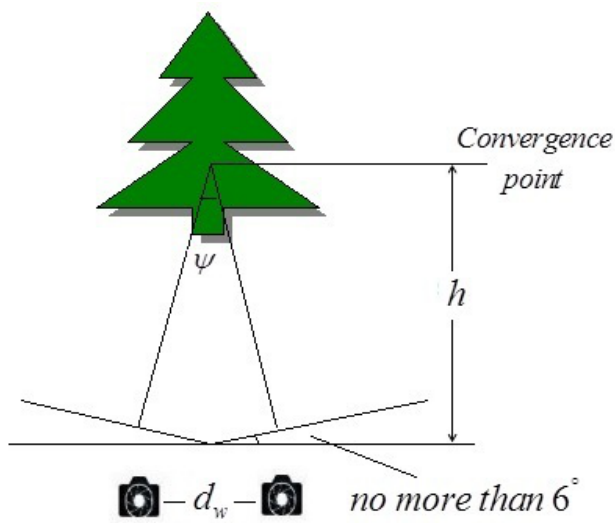

Fig. $1.12^{\circ}$ theory schematic diagram

- $1 / 30$ rule: in professional stereo shooting activities, the $1 / 30$ rule of thumb of 3D [20]-[22], which stipulates that the inter-camera distance should be $1 / 30$ of the distance from the camera to the first foreground object, is widely used in stereo photography.

- $12^{\circ}$ theory: for toed-in camera configuration, $12^{\circ}$ theory [7] can control the range of inter-camera distance in all scales. The $12^{\circ}$ theory means the incline angle of the camera does not exceed $6^{\circ}$ in macro shooting, at the same time the convergence angle $(\psi)$ of camera optical axes is not more than $12^{\circ}$ (shown in Fig. 1). The theory is shown in Eq. 1.

$$
d_{w}=2 \cdot h \cdot \tan \left(\frac{\psi}{2}\right)
$$

where $d_{w}$ is the theoretical inter-camera distance, $h$ is the shooting distance, $\psi \leq 12^{\circ}$.

- Ratio of binocular overlap to visual field: the magnification of an image on the retina is $B E / C E$ [13], [15], [23], [24], shown in Fig. 2 (here, $B E$ is the width of captured stereo image [25], $C E$ is the width of composite image, denoting the binocular overlap of stereo camera). The value of $B E / C E$ can affect the values of the positive and the negative parallax and further affect the quality of the stereo images. To simplify the calculation, specify $C E / B F$ as the evaluation index in this paper, where $B F$ is the camera viewing region. The effect of stereo images varies with the change of $C E / B F . p$ is the viewing angle of the stereo camera (as shown in Table I). Based on the geometric relationship in Fig. 2, we make the conclusion present in Eq. 2.

$$
\left\{\begin{array}{r}
\frac{C E}{B F}=\frac{C E}{B C+C E+E F} \\
B C=E F=d \\
B E=B C+C E \\
h=\frac{B E / 2}{\tan (p / 2)}
\end{array}\right.
$$

- Angular disparity theory: studies about human factors suggested that a certain angular disparity should be maintained in order to generate comfortable images [24]. Previous study indicated that, if the angular disparity $\xi$ equals to $70^{\prime}$ [8], the better stereo effect can be achieved without visual discomfort. In Fig. 2, $\alpha$ is the angle on the convergent direction, and $\beta$ 


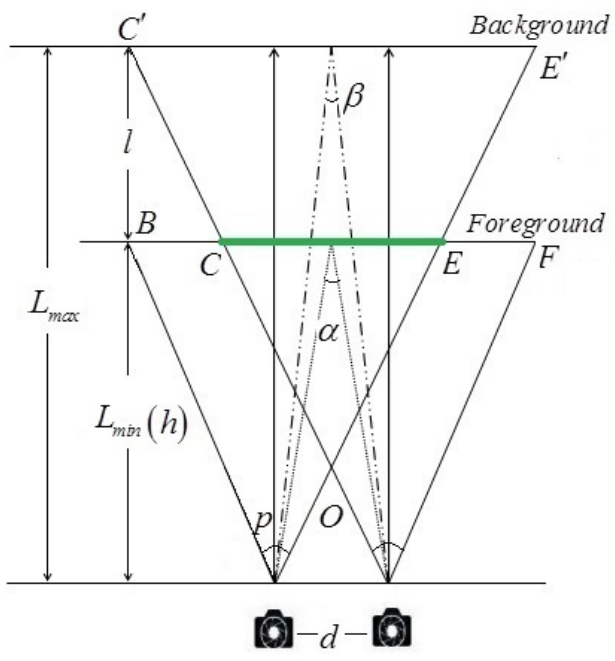

Fig. 2. Binocular overlap and angular disparity schematic diagram for parallel camera configuration

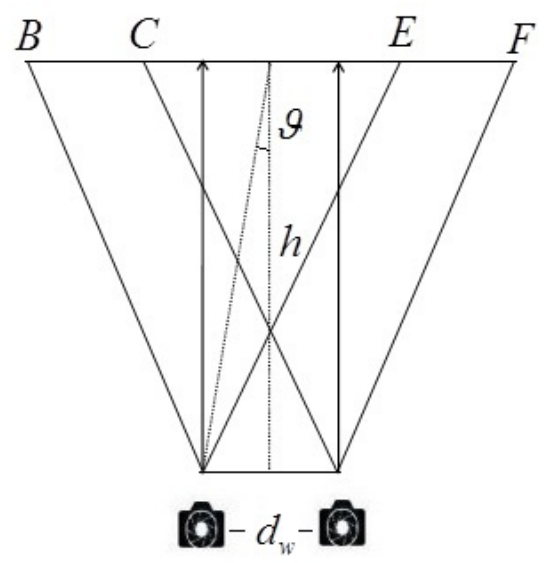

Fig. 3. Visual acuity of camera schematic diagram

is the angle on the divergence direction. The corresponding relationship among $\alpha, \beta$ and $\xi$ is shown in Eq. 3 .

$$
\left\{\begin{array}{r}
\alpha=2 \cdot \arctan \left(\frac{d / 2}{L_{\min }}\right) \\
L_{\max }=L_{\min }+l \\
\beta=2 \cdot \arctan \left(\frac{d / 2}{L_{\max }}\right) \\
\xi=\alpha-\beta
\end{array}\right.
$$

where, $d$ is inter-camera distance, $L_{\min }$ is the distance from the closest scene to the camera center, $L_{\max }$ is the distance from the farthest scene to the camera center.

- Visual acuity of camera theory: the visual acuity of a camera, $\vartheta$ shown in Fig. 3, is widely recognized as $0.5^{\circ}$ [26]. If the shooting distance $h$ is known, we can get the theoretical inter-camera distance $d_{w}$ according to the visual acuity of the camera,

$$
d_{w}=2 \cdot h \cdot \tan (\vartheta)
$$

- Ratio of stereo parallax to image width: previous studies [27] investigated the effect of CCD size $W$ and the width
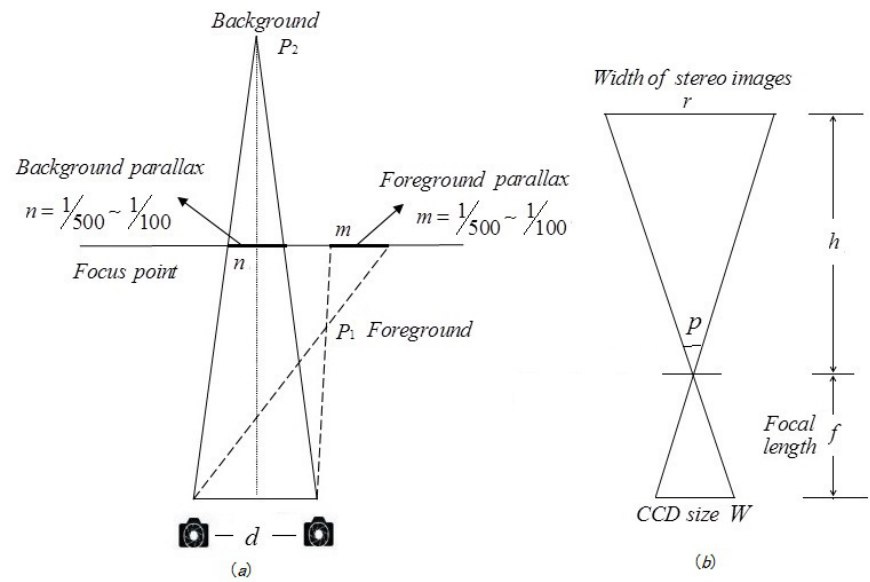

Fig. 4. (a) Foreground and background parallax range schematic diagram; (b) Camera internal structure schematic diagram

of stereo images $r$ on the parallax, they also concluded that the stereoscopic effect of images is better when the range of 3D image parallax within $r / 500-r / 100$ ( shown in Fig. 4 (a)) than other conditions. $r$ can be obtained according to the geometric relationship between the parameters in Fig.4 (b), as shown in Eq. 5.

$$
\left\{\begin{array}{r}
r=\frac{h}{f} \cdot W \\
r=2 \cdot h \cdot \tan \left(\frac{p}{2}\right)
\end{array}\right.
$$

The existing shooting principles are simple two-level criteria, which means that the score of shooting quality according to each principle is either good or not, and cannot meet the fivelevel evaluation demand. Previous proposed stereo shooting principles or models generally take part of influenced factors into consideration or based on human perception, also the shooting principles are two-level evaluation criteria. In 2000, Yamanoue et al. based on a five-point scale of subjective perception, studied the relationship between shooting condition and cardboard effect of stereoscopic images [10]. Later, they used the five-point scale as the evaluation level in the subjective experiment in the work [15], and did the geometrical analysis of puppet-theater and cardboard effects. In this paper, as in [10] and [15], we adopt the five-point scale to instruct the observers to rate the quality of the stereo images from 1 to 5 . The proposed five-level subjective evaluation standard for stereo cameras is shown in Table II.

\section{ObJective Shooting Quality Evaluation CRITERIA For Stereo CAMERAS}

Based on the previous studies on shooting principles and the characteristics of two types of stereo cameras [16], [17], we analyze two objective evaluation criteria of shooting quality for parallel and toed-in camera configurations, respectively, under three shooting conditions (macro shooting, short and long distance shooting). Then the criteria are integrated into an overall quality index. The frameworks of the proposed criteria are shown in Fig. 5 and Fig. 6. 
TABLE II

Criteria For SubJective Quality EVALUATION OF STEREo CAMERA

\begin{tabular}{|c|c|c|}
\hline Response & Explanation & Quality \\
\hline 5 & $\begin{array}{l}\text { Imperceptible: there is no any } \\
\text { damage on depth perception, } \\
\text { and image quality, looks comfor- } \\
\text { table and natural, suitable for } \\
\text { human visual experience. }\end{array}$ & Excellent \\
\hline 4 & $\begin{array}{l}\text { Perceptible but not annoying: } \\
\text { there is a slight loss on } \\
\text { depth perception, but the quality } \\
\text { of the whole image is still good, } \\
\text { suitable for human visual experience. }\end{array}$ & Good \\
\hline 3 & $\begin{array}{l}\text { Slightly annoying: there is obvious } \\
\text { loss on depth perception; how- } \\
\text { ever you can accept this } \\
\text { quality, reluctantly, generally suit- } \\
\text { able for human visual experience. }\end{array}$ & Fair \\
\hline 2 & $\begin{array}{l}\text { Annoying: there is need to carefully } \\
\text { distinguish the depth perception } \\
\text { not suitable for visual experience. }\end{array}$ & Poor \\
\hline 1 & $\begin{array}{l}\text { Very annoying: nearly no depth } \\
\text { perception, people feel uncomfortable. }\end{array}$ & $\mathrm{Bad}$ \\
\hline
\end{tabular}

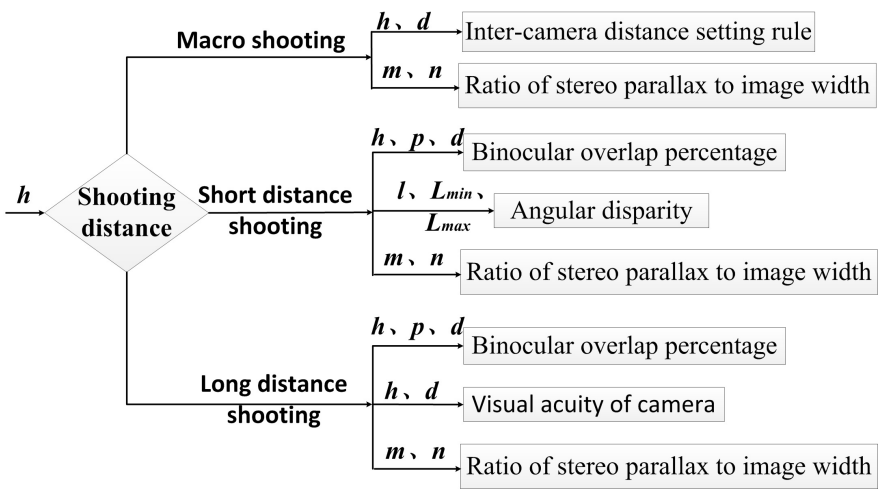

Fig. 5. Objective criterion for shooting quality evaluation of parallel camera configuration

\section{A. Explanation of the Objective Evaluation Criteria}

In order to explain the ideas underline the shooting quality evaluation criteria frameworks, we first explain how to build the criteria shown in Fig. 5 and Fig. 6. In 2003, Lao et al. [27] presented that image parallax plays an important role in shooting quality for both parallel and toed-in camera configurations. So we take "Ratio of stereo parallax to image width" as one of the key factors under all the shooting conditions for both types of stereo cameras. What's more, it is worth mentioning that compared with the toed-in camera configuration, parallel camera configuration has an unique and special area called the binocular overlap area, which means: no matter how other shooting parameters change, the inter-camera distance always equal to the horizontal shift between the right and left images. Based on the binocular overlap area, the factor of "Binocular overlap percentage" is applied to the criteria of short and long distance shooting of parallel camera configuration, but not macro shooting condition. Because of the limit shooting distance, the factor of "Binocular overlap percentage" has little effect on the quality of macro shooting condition. Besides, because of the complicated calculating process, "Binocular overlap percentage" is not used to build the criteria of toed-

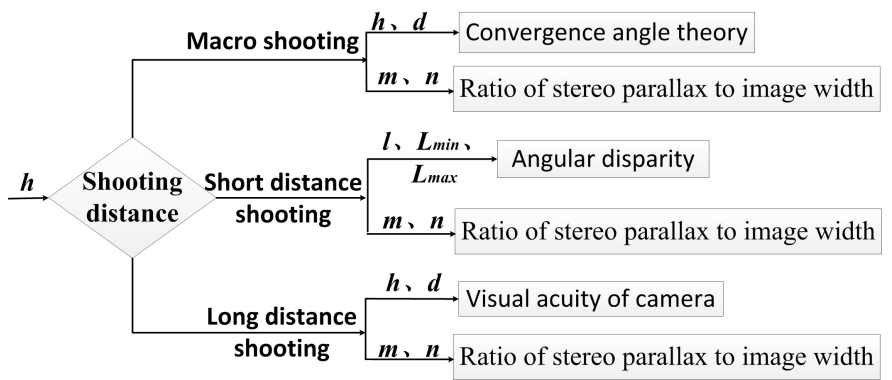

Fig. 6. Objective criterion for shooting quality evaluation of toed-in camera configuration

in camera configuration. The specific shooting criteria are explained as follows:

Parallel camera configuration: the parallel camera configuration converges at infinity and the captured $3 \mathrm{D}$ scene appears to be entirely in front of the screen. And each photographed scene is known to have a negative horizontal pixel parallax [28]. For macro shooting, because of small shooting area, the foreground parallax is important in the shooting quality. So we add "Inter-camera distance setting rule" [20]-[22] to reflect the effect of foreground parallax. For short distance shooting, the scale of shooting scene is bigger than macro shooting, thus "Angular disparity" [8], [24] which determine the whole parallax distribution is selected to evaluate shooting quality. Then "Binocular overlap percentage" is also considered to be one of shooting principles under both short and far distance shooting. For far distance shooting, because of the limited visual acuity of stereo camera [26], the background stereo shooting quality is very important. Therefore, in addition to "Binocular overlap percentage" and "Ratio of stereo parallax to image width", we further add "Visual acuity of camera" to establish the parallel shooting criteria.

Toed-in camera configuration: different from parallel camera configuration, toed-in camera configuration converges at a single point and the shooting images obtain the concaveconvex feelings [7]. These different structure characteristics lead to different evaluation criteria. For macro shooting, the foreground parallax is determined by "Convergence angle theory" which is selected as one shooting criterion. For short distance shooting, without the characteristic of "Binocular overlap percentage", we apply "Angular disparity" for short distance shooting quality evaluation criteria. For far distance shooting, "Visual acuity of camera" is used for far distance shooting quality evaluation.

In summary, all the corresponding individual influencing factors of each shooting condition and the comparison between the proposed method and the existing methods are shown in Table III.

\section{B. Evaluation Experiments and Establishment for Each $\mathrm{Ob}$ - jective Evaluation Criterion}

This section aims to introduce the evaluation experiments to obtain the subjective and objective assessment scores, and present individual influencing factors of parallel and toed-in camera configurations, respectively. 
TABLE III

Components of Proposed Criteria in this Paper Including All the Shooting Principles Studied in the Literature and the Proposed Evaluation Theories for Stereo Camera in this Paper. Here "Existing" Means the Previous Shooting Principles in Literature, and "Proposed” is the Proposed Five-Level Evaluation Criterion in this Paper

\begin{tabular}{|c|c|c|c|}
\hline Shooting type & Distance & Evaluation factor & Comparison \\
\hline \multirow[b]{3}{*}{ Parallel } & \multirow{2}{*}{$\begin{array}{l}\text { Macro } \\
\text { shooting }\end{array}$} & $\begin{array}{l}\text { Inter-camera distance } \\
\text { setting rule }\end{array}$ & $\begin{array}{c}\text { Existing: when } d / h=1 / 30 \text {, good stereoscopic effect, } \\
\text { [20]-[22] it is a two-level evaluation criterion } \\
\text { Proposed: with the range of } d / h \text {, present a five-level } \\
\text { evaluation criterion (Section IV.A.1)) }\end{array}$ \\
\hline & & $\begin{array}{l}\text { Ratio of stereo } \\
\text { parallax to } \\
\text { image width }\end{array}$ & $\begin{array}{l}\text { Existing: stereoscopic effect of images is good when } \\
\text { the range of } 3 \mathrm{D} \text { image parallax was within } r / 500-r / 100 \text { [27] } \\
\text { Proposed: named } c \text { and } c_{0} \text { as ratio of total and fore- } \\
\text { ground parallax, respectively, present a five- } \\
\text { level evaluation criterion (Section IV.A.1)) }\end{array}$ \\
\hline & \multirow{3}{*}{$\begin{array}{l}\text { Short } \\
\text { distance } \\
\text { shooting }\end{array}$} & $\begin{array}{l}\text { Binocular overlap } \\
\text { percentage }\end{array}$ & $\begin{array}{l}\text { Existing: value of } B E / C E \text { (Fig. 2), can affect the stereo- } \\
\text { scopic effect of the captured images [13], [15], [23], [24] ) } \\
\text { Proposed: specify } C E / B F \text { as the evaluation index } \\
\text { to explore the factor effect rule (Section IV.A.2)) }\end{array}$ \\
\hline \multirow{5}{*}{ configuration } & & Angular disparity & $\begin{array}{c}\text { Existing: good stereo effect can be achieved, } \\
\text { when } \xi \text { equals to } 70^{\prime}[24] \\
\text { Proposed: establish a five-level evaluation factor } \xi \\
\text { through experiments probe mapping between } \\
\xi \text { and MOS value (Section IV.A.2)) }\end{array}$ \\
\hline & & $\begin{array}{l}\text { Ratio of stereo parallax } \\
\text { to image width }\end{array}$ & $\begin{array}{c}\text { Ranges of } c \text { and } c_{0} \text { are similar to } \\
\text { those under macro shooting }\end{array}$ \\
\hline & \multirow{3}{*}{$\begin{array}{c}\text { Long } \\
\text { distance } \\
\text { shooting }\end{array}$} & $\begin{array}{l}\text { Binocular overlap } \\
\text { percentage }\end{array}$ & $\begin{array}{l}\text { Factor affect rule is same with } \\
\text { that under macro shooting }\end{array}$ \\
\hline & & $\begin{array}{l}\text { Visual acuity } \\
\text { of camera }\end{array}$ & $\begin{array}{l}\text { Existing: it is widely recognized as } 0.57^{\circ} \text { [26], people } \\
\text { can get } d_{w} \text { in case of the known } h \\
\text { Proposed: with range of visual acuity, we establish } \\
\text { a five-level evaluation criterion (Section IV.A.3)) }\end{array}$ \\
\hline & & $\begin{array}{l}\text { Ratio of stereo parallax } \\
\text { to image width }\end{array}$ & $\begin{array}{l}\text { Ranges of } c \text { and } c_{0} \text { are similar to } \\
\text { those under macro shooting }\end{array}$ \\
\hline \multirow{3}{*}{ Toed-in } & \multirow[t]{2}{*}{$\begin{array}{l}\text { Macro } \\
\text { shooting }\end{array}$} & $\begin{array}{l}\text { Convergence angle } \\
\text { theory }\end{array}$ & $\begin{array}{l}\text { Existing: convergence angle is not more than } 12^{\circ} \\
\text { it is a two-level evaluation criterion } \\
\text { Proposed: a corresponding five-level convergence angle } \\
\text { theory is established for extensive utilization (Section IV.B.1)) }\end{array}$ \\
\hline & & $\begin{array}{l}\text { Ratio of stereo parallax } \\
\text { to image width }\end{array}$ & $\begin{array}{l}\text { Ranges of } c \text { and } c_{0} \text { and those of parallel } \\
\text { camera configuration are the same }\end{array}$ \\
\hline & \multirow{2}{*}{$\begin{array}{c}\text { Short } \\
\text { distance } \\
\text { shooting }\end{array}$} & $\begin{array}{l}\text { Angular } \\
\text { disparity }\end{array}$ & $\begin{array}{l}\text { Similar to the parallel camera configuration, we will } \\
\text { establish a corresponding five-level establish criterion }\end{array}$ \\
\hline camera & & $\begin{array}{l}\text { Ratio of stereo parallax } \\
\text { to image width }\end{array}$ & $\begin{array}{c}\text { Ranges of } c \text { and } c_{0} \text { and those of parallel } \\
\text { camera configuration are the same }\end{array}$ \\
\hline \multirow[t]{2}{*}{ configuration } & \multirow{2}{*}{$\begin{array}{c}\text { Long } \\
\text { distance } \\
\text { shooting }\end{array}$} & $\begin{array}{l}\text { Visual acuity } \\
\text { of camera }\end{array}$ & $\begin{array}{l}\text { Similar to the parallel camera configuration, we will } \\
\text { establish a corresponding five-level establish criterion }\end{array}$ \\
\hline & & $\begin{array}{l}\text { Ratio of stereo parallax } \\
\text { to image width }\end{array}$ & $\begin{array}{l}\text { Ranges of } c \text { and } c_{0} \text { and those of parallel } \\
\text { camera configuration are the same }\end{array}$ \\
\hline $\begin{array}{l}r: \text { the image wid } \\
\vartheta: \text { the visual acui } \\
\xi: \text { the index of a } \\
k_{w}: \text { the converge } \\
v: \text { the total parall } \\
C E / B F: \text { the in } \\
d / h: \text { index of int } \\
c_{0}: \text { the index of } \\
c: \text { the index of ra } \\
k: \text { the index of v } \\
k_{f}: \text { the index of } \\
Q: \text { the comprehe }\end{array}$ & $\begin{array}{l}\text { y of camera; } \\
\text { gular disparit } \\
\text { ce angle the } \\
x \text { of stereo i } \\
\text { ex of binocu } \\
\text { r-camera dis } \\
\text { tio between } \\
\text { lo between f } \\
\text { ual acuity of } \\
\text { isual acuity } \\
\text { sive objective }\end{array}$ & $\begin{array}{l}\text { y; } \\
\text { ages; } \\
\text { ir overlap ratio; } \\
\text { nce setting rule; } \\
\text { tal parallax and image width; } \\
\text { eground parallax and image w } \\
\text { camera for parallel camera cor } \\
\text { camera for toed-in camera cc } \\
\text { evaluation criteria. }\end{array}$ & $\begin{array}{l}\text { dth; } \\
\text { iguration; } \\
\text { figuration; }\end{array}$ \\
\hline
\end{tabular}

\section{1) Evaluation experiments:}

a) Participants: fifty non-professional adults, age from 21 to 36 and all have normal stereo acuity with binocular vision above 0.8 , participate in the subjective assessments. Before the subjective experiments, they are asked to take the binocular visual color test and stereo vision test (by viewing the synoptophore or stereo visual inspection pictures).

b) Apparatus: 3D display is the most common media that people watch stereoscopic image and percept the shooting quality, so the display aspects, e.g. display size, watching condition, should not be overlooked [16]. It is important to acknowledge that the depth perceived in stereoscopic content is strongly linked to the characteristics of 3D display such as the size of the display screen [9], viewing condition and so on. However, it is a big topic if we add all of the factors, so the effect of other parameters on shooting quality will be studied in the future. Here, in order to avoid the effect of viewing condition, the observers in the subjective experiments are all suggested to conduct the experiments at the comfortable viewing range suggested by the instructions of each display 

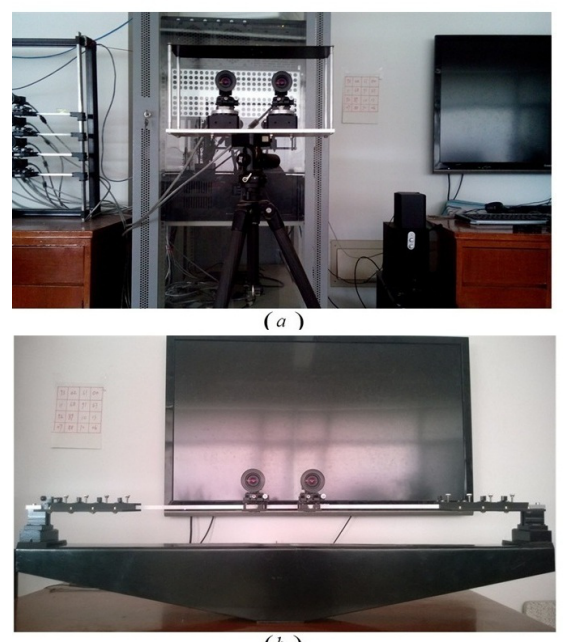

(b)

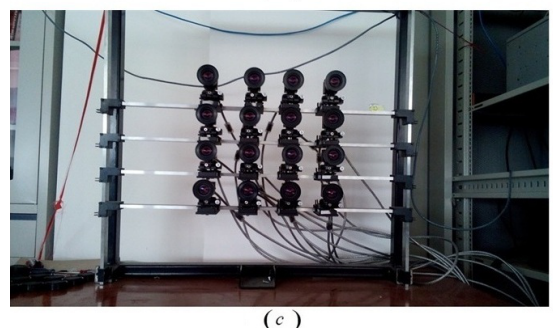

(c)

Fig. 7. Real stereo cameras schematic diagram. Sony ICX445 CCD, 1/3", $3.75 \mu \mathrm{m}$; Global Shutter; $1024 \times 768$ at 30 FPS. (a) Inter-camera distance can be changed to get macro and short distance shooting images, and also, the parallel and toed-in camera configurations can be obtained; $(b)$ Inter-camera distance can be changed to get macro, short and long distance shooting images, and also the parallel and toed-in camera configurations can be obtained; can get bigger inter-camera distance; $(c)$ Matrix multi-camera arrangement.

device. The subjective tests in this paper are conducted on three different sizes of stereoscopic displays, namely, Philips 423D6W0200 42 inches multi-view auto stereoscopic display, Hyundai S465D 46 inches 3D stereoscopic LCD display and LG 47CM540-CA 47 inches 3D HDTV display. The Philips stereoscopic display can be viewed without glasses and the rest two are paired with their own 3D Active glasses.

c) Source images: double viewpoint images are taken for the subjective experiments. These stereo images are extracted from the stereo image library in the stereo vision laboratory of School of Electronic Information Engineering, Tianjin University, and these images are not selected randomly but according to the stereo scene and shooting parameters. The stereo images in this library are captured by Autodesk 3ds Max and stereo cameras in the laboratory (shown in Fig. $7(a)-(c)$ ). The size of the training and test stereo images is $1024 \times 768$. This database consists of 3636 stereoscopic pairs under various shooting conditions: macro shooting, short distance shooting, and long distance shooting (as shown in Table IV).

d) Procedure: before formal experiments, all participants watch randomly ordered training stereo images for 8 seconds at a viewing distance which is equal to the height of the screen multiplied by factor 3 as suggested in the ITU-R BT.1438 for HDTV [29], then they are asked to evaluate the stereo images with different camera parameters. For example, for the stereo images under macro shooting which are captured with
TABLE IV

Numbers of STEREO IMAgES UNDER VARIOUS SHOOTING CONDITIONS

\begin{tabular}{c|cccc}
\hline Sype & Macro & Short & Long & All \\
\hline Parallel camera configuration & 935 & 520 & 365 & 1820 \\
\hline Toed-in camera configuration & 932 & 520 & 364 & 1816 \\
\hline All & 1867 & 1040 & 729 & 3636 \\
\hline
\end{tabular}

different camera parameters while the other parameters keep their best value, the observers watch each of the randomly ordered stereo images for $8 \mathrm{~s}$ followed by a $5 \mathrm{~s}$ interval, so the subjects have enough time to make the right response and 5 s interval can guarantee that each evaluation value is not affected by memory effect [30]. For each of the durations, observers are asked to rate the quality of stereo images using the five-point scale, as shown in Table II. In our experiments, participants are allowed to take a break (e.g. 10 minutes in our experiment) after every 25 minutes quality assessment. The specific time intervals in this paper are determined based on our experience, feedbacks from our pilot studies and previous studies [14][31][32][33]. For the other shooting conditions, we adopt the same procedure above.

The mean opinion score (MOS) [34] is firstly computed for each image by averaging all the subjects' scores, and the Student's t-test [35] is adopted to compute confidence intervals with the significant level being 95\%. Then we calculate the range of each influenced factors, and summarize the mapping between each factor and MOS value. The same processes are repeated for the establishment of short and long distance shooting evaluation criteria.

\section{ESTABLISHMENT OF SHOOTING PRINCIPLES AND EVALUATION THEORIES}

Based on the shooting principles described in Section II, in this section we extend the existing principles (called "Existing" in Table III) to a five-level criteria (called "Proposed" in Table III) and propose new shooting principles of five-level criteria through experiments. The details of each proposed principle are presented in the following sections.

\section{A. Shooting Principles and Evaluation Theory for Parallel Camera Configuration}

Based on the subjective experimental results, we establish the five-level mapping between individual factors and MOS value for each shooting distance, as shown in Table V. The specific experiments conduction and explanations are presented as follows:

1) Macro shooting principles: The evaluation of macro shooting is studied using the inter-camera distance setting rule and the ratio of stereo parallax to image width.

Inter-camera distance setting rule: $d / h$ is specified as the evaluation index of the inter-camera distance setting rule. Based on a series of stereoscopic image pairs and $d / h$ ranges from $1 / 80$ to $1 / 5$, the five-level mapping between the intercamera distance and MOS value is built through associating subjective experimental results with the range of $d / h$ value shown in Table V. The results indicate that the value $1 / 30$ 


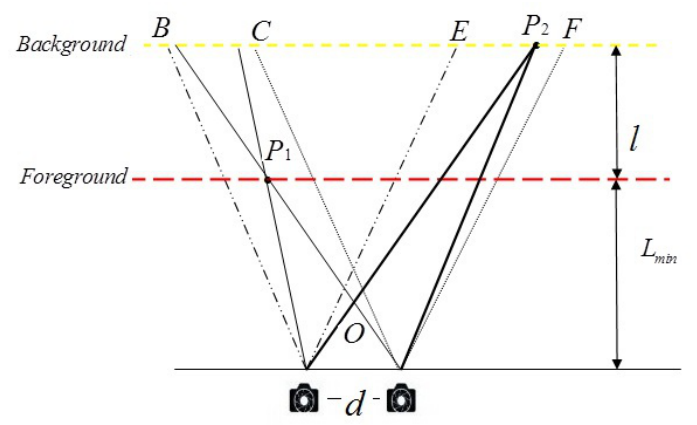

Fig. 8. Total parallax schematic diagram of parallel camera configuration

proposed before [20-22] cannot precisely evaluate the shooting quality, and the specific threshold should be 1/50. To get much better image quality, $d / h$ should be set less than $1 / 75$. Fivelevel evaluation criterion provides multiple choices to set the shooting parameters $\mathrm{d}$ and $\mathrm{h}$, and help people get what they really want (shooting quality ranges from 1 to 5), while the two-level evaluation cannot.

Ratio of stereo parallax to image width: stereo image parallax is an important factor which affects stereo image quality. Although the setting rule of the parallax is already proposed by Lao et al. [27], but it is a two-level evaluation principle, so it cannot be directly used in our criteria. Therefore, a corresponding five-level evaluation criterion needs to be created. This paper takes the ratio of stereo parallax of image width as the final evaluation index to establish the evaluation criteria. For parallel camera configuration (shown in Fig. 8), the stereo images display the shooting scene just like in front of the display screen, the foreground and the background deviate to the same direction. The foreground parallax is greater than the background parallax, so horizontal total parallax of stereo images is the difference between foreground and background parallax [27].

The ratio of total parallax to image width $c_{0}$ can be obtained from Eq. 6:

$$
\left\{\begin{array}{r}
c_{0}=\frac{v}{r} \\
v=m-n
\end{array}\right.
$$

where $r$ is the horizontal width of stereo images, $v$ is the total parallax of stereo images [27], $m$ is the foreground parallax of stereo images and $n$ is the background parallax of stereo images.

Besides, the foreground parallax has a great effect on the visual comfort and the stereoscopic effect. The ratio of the foreground parallax to image width $c$ (shown in Eq. 7) needs to be taken into consideration.

$$
c=\frac{m}{r}
$$

In our experiments, a series of different foreground parallax and background parallax are involved. The value of $c$ ranges from 0 to 5.72, and $c_{0}$ ranges from 0 to 3.24 , the mapping between MOS and $c$ as well as $c_{0}$ is further obtained from the experiments as shown in Table $\mathrm{V}$.
2) Short distance shooting principles: The evaluation of stereo camera for short distance shooting is conducted based on the binocular overlap percentage, angular disparity and the ratio of stereo parallax to image width.

Binocular overlap percentage: according to the experiments, we find that the size of the binocular overlap has a certain influence on the stereo image quality. Therefore, this paper establish an evaluation criterion which regards the ratio of binocular overlap to visual field as an evaluation criterion. The individual factor $C E / B F$ is taken as the evaluation index. All test images are divided into several different groups, where the $C E / B F$ value ranges from 0.85 to 0.9985 . Through the experiments, we obtain the mapping between $C E / B F$ and MOS value, as shown in Table V.

Angular disparity: in our experiments, a set of stereo images with different angular disparity $\xi$ (shown in Eq. 3), ranges from $0^{\prime}$ to $91.8^{\prime}$, are used to establish the five-level evaluation criterion. The mapping between $\xi$ and MOS value indicates that when $\xi$ value is not more than $71.09^{\prime}$, people can get a good stereoscopic effect. Different from the value of $70^{\prime}$ in [8], when $\xi$ is set to $71.09^{\prime}$, people also can obtain the good stereoscopic images. The previous two level evaluation criterion limits the range of shooting parameters and may cause some shooting problems (i.e. increase shooting difficulty and shooting cost).

Ratio of stereo parallax to image width: based on our experimental results, the evaluation mapping is same with that for macro shooting as shown in Table V. With the increasing of $c$ and $c_{0}$, the corresponding MOS value is decreasing, the smaller the value of $c$ and $c_{0}$, the better the stereoscopic effect.

3) Long distance shooting principles: The evaluation for stereo camera long distance shooting is conducted based on the binocular overlap percentage, visual acuity of camera and influence of the ratio of stereo parallax to image width.

Binocular overlap percentage: it is one of the significant factors which has great effect on long distance shooting quality. The mapping between binocular overlap percentage and MOS value shows that when $C E / B F$ is not more than 0.9596 , there is a bit loss on depth perception and this will cause an uncomfortable stereo effect.

Visual acuity of camera: in long distance shooting, the visual acuity of camera is the main factor. Ignoring this factor could result in viewing uncomfortable or even loss of stereo impression. Here let $k$ denotes $\tan (\vartheta)$, the value of $k$ ranges from 0 to 0.05 . The mapping between $k$ and MOS value is shown in Table V, the shooting quality will be better when it has a lower $k$ value, no more than 0.013 .

Ratio of stereo parallax to image width: through experiments, we find that the evaluation criterion is the same as that of macro and short distance shooting. It indicates that this factor is appropriate for any shooting distance.

\section{B. Shooting Principles and Evaluation Theory for Toed-in Camera Configuration}

Like parallel camera configuration, we also summarize the five-level mapping for each case of toed-in camera configuration, as shown in Table VI, which are explained as follows: 
TABLE V

MAPPING BETWEEN INDIVIDUAL FACTORS AND MOS VALUE OF PARALLEL CAMERA CONFIGURATION

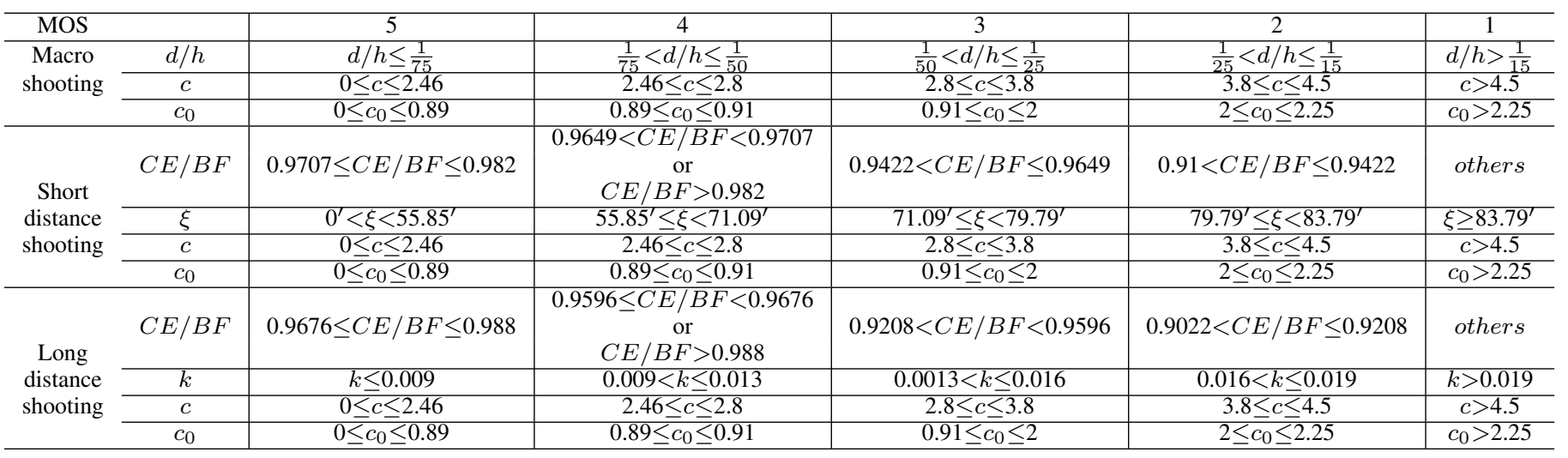

TABLE VI

MAPPING BETWEEN INDIVIDUAL FACTORS AND MOS VALUE OF TOED-IN CAMERA CONFIGURATION

\begin{tabular}{cc|c|c|c|c|c}
\hline MOS & & 5 & 4 & 3 & 2 & 1 \\
\hline \multirow{2}{*}{$\begin{array}{c}\text { Macro } \\
\text { shooting }\end{array}$} & $k_{w}$ & $k_{w} \leq 0.069$ & $0.069<k_{w} \leq 0.075$ & $0.075<k_{w} \leq 0.105$ & $0.105<k_{w} \leq 0.125$ & $k_{w}>0.125$ \\
\cline { 2 - 7 } & $c$ & $0 \leq c \leq 2.46$ & $2.46 \leq c \leq 2.8$ & $2.8 \leq c \leq 3.8$ & $3.8 \leq c \leq 4.5$ & $c>4.5$ \\
\cline { 2 - 7 } & $c_{0}$ & $0 \leq c_{0} \leq 0.89$ & $0.89 \leq c_{0} \leq 0.91$ & $0.91 \leq c_{0} \leq 2$ & $2 \leq c_{0} \leq 2.25$ & $c_{0}>2.25$ \\
\hline \multirow{2}{*}{$\begin{array}{c}\text { Short } \\
\text { distance } \\
\text { shooting }\end{array}$} & $\xi$ & $0^{\prime}<\xi<55.85^{\prime}$ & $55.85^{\prime} \leq \xi<71.09^{\prime}$ & $71.09^{\prime} \leq \xi<79.79^{\prime}$ & $79.79^{\prime} \leq \xi<83.79^{\prime}$ & $\xi \geq 83.79^{\prime}$ \\
\cline { 2 - 7 } & $c$ & $0 \leq c \leq 2.46$ & $2.46 \leq c \leq 2.8$ & $2.8 \leq c \leq 3.8$ & $3.8 \leq c \leq 4.5$ & $c>4.5$ \\
\hline \multirow{2}{*}{$\begin{array}{c}\text { Long } \\
\text { distance } \\
\text { shooting }\end{array}$} & $0 \leq c_{0} \leq 0.89$ & $0.89 \leq c_{0} \leq 0.91$ & $0.91 \leq c_{0} \leq 2$ & $2 \leq c_{0} \leq 2.25$ & $c_{0}>2.25$ \\
\cline { 2 - 7 } & $k_{f}$ & $0<k_{f} \leq 0.03$ & $0.03<k_{f} \leq 0.05$ & $0.05<k_{f} \leq 0.06$ & $0.06<k_{f} \leq 0.07$ & $k_{f}>0.07$ \\
\cline { 2 - 7 } & $c$ & $0 \leq c \leq 2.46$ & $2.46 \leq c \leq 2.8$ & $2.8 \leq c \leq 3.8$ & $3.8 \leq c \leq 4.5$ & $c>4.5$ \\
\hline
\end{tabular}

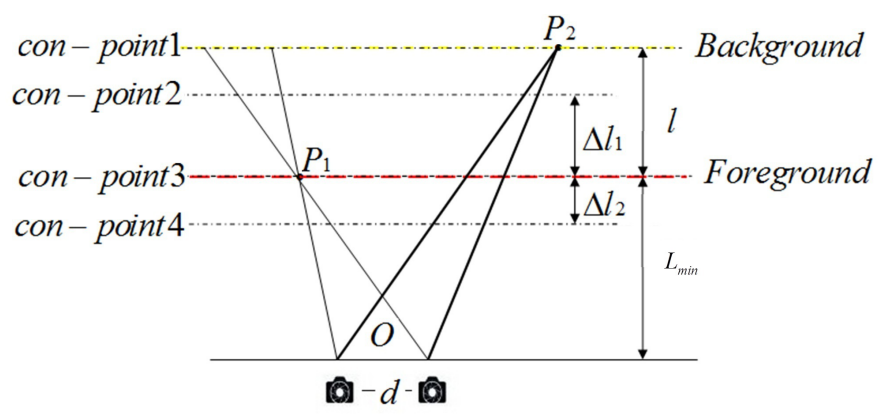

Fig. 9. Total parallax schematic diagram of toed-in camera configuration

1) Macro shooting principles: To derive the evaluation of macro shooting for toed-in camera configuration, the following factors are taken into account: convergence angle theory and the ratio of stereo parallax to image width.

Convergence angle theory: in order to establish the fivelevel convergence angle theory, here let $k_{w}$ denotes $\tan \left(\frac{\psi}{2}\right)$ ( $\psi$ shown in Fig. 1), with the value of $k_{w}$ in our experiments ranges from 0 to 0.3 . According to the subjective experiments, mapping between $k_{w}$ and MOS value is established, which is suitable for evaluating the influence of inter-camera distance on the stereo image quality in the macro shooting of toedin camera configuration. Based on the previous paper in [7], convergence angle should be set no more than $12^{\circ}$, i.e. $k_{w}$
TABLE VII

TOTAL PARALLAX $v$ OF DIFFERENT CONVERGENCE POINT

\begin{tabular}{c|c}
\hline The convergence point, shown in Fig. 9 & The total parallax $v$ \\
\hline On the background, (con-point 1) & $v=m$ \\
In the middle of a scene, (con-point 2) & $v=m+n$ \\
On the foreground, (con-point 3) & $v=n$ \\
In front of the foreground, (con-point 4) & $v=n-m$
\end{tabular}

should be less than 0.1051. However, our results shown in Table VI indicate that $k_{w}$, ranges from 0.075 and 0.105 , leads to bad quality of stereoscopic image, and the evaluation rate is 3 . The above results indicate that the five-level evaluation criterion is more effective to assess the shooting quality than two level evaluation criterion.

Ratio of stereo parallax to image width: different from parallel camera configuration, here when the position of the convergence point is changed, the total parallax $v$ of stereo images is different, as shown in Fig. 9 and Table VII, which is analyzed from four aspects based on the location of the convergence point.

$c_{0}$ and $c$ can be calculated from Eq. 6 and Eq. 7. Through experiments, series of stereo image pairs are captured with different foreground parallax and background parallax. The value of $c$ ranges from 0 to 5.72, and $c_{0}$ ranges from 0 to 3.24. Based on the subjective evaluation experiment results, we obtain the mapping among $c, c_{0}$ and MOS value, as shown in Table VI, and it is same with the mapping in macro shooting for parallel camera configuration. 


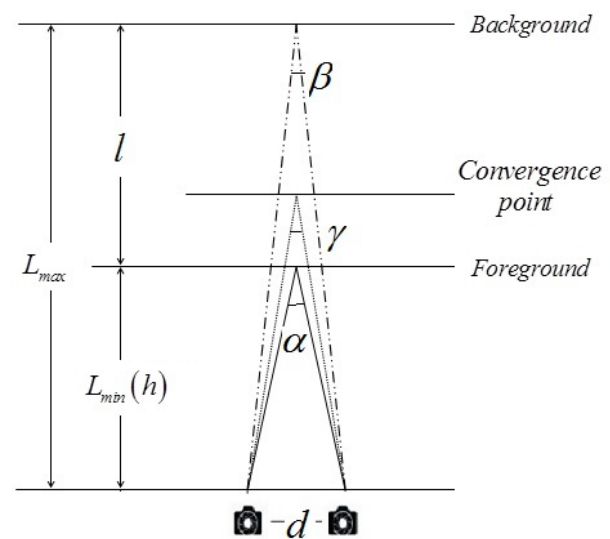

Fig. 10. Angular disparity schematic diagram of toed-in camera configuration

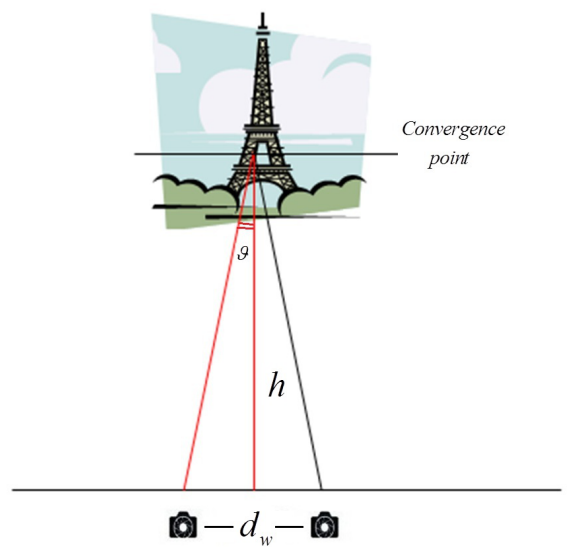

Fig. 11. Visual acuity of camera schematic diagram

2) Short distance shooting principles: The following experiments is conducted.

Angular disparity: the principle of angular disparity is also adapted to the short distance shooting of the toed-in camera configuration, shown in Fig. 10. With a common value of $h$, another set of stereo images are captured by changing the value of $d . \alpha, \beta$ and $\xi$ can be obtained according to Eq. 3 . Then we change the value of $h$ to get another series of stereo images. The value of angular disparity $\xi$ ranges from $0^{\prime}$ to 93'. Subjective experimental results show that the evaluation criterion of toed-in camera configuration is same with that of parallel camera configuration short distance shooting (shown in Table VI).

Ratio of stereo parallax to image width: similar experiments are carried out under the same condition of the macro shooting of parallel camera configuration, we find that the two evaluation criteria are the same for the two camera configurations. With the decreasing of $c$ and $c_{0}$ value, people can get a better stereoscopic effect.

3) Long distance shooting principle: In long distance shooting, the evaluation is done from two aspects: visual acuity of camera and the ratio of stereo parallax to image width.

Visual acuity of camera: in long distance shooting, the visual acuity of camera $\vartheta$ (as shown in Fig. 11) plays an important role in image quality assessment. To establish a five-level evaluation criteria based on the visual acuity of camera, a series of experiments are done, and the obtained mapping between $k_{f}$ and MOS value is shown in Table VI. Here specify $\tan (\vartheta)$ as $k_{f}$, ranges from 0 to 0.2 . The final evaluation factor $k_{f}$ about visual acuity of camera is presented based on these experimental results.

Ratio of stereo parallax to image width: through the experiment results, the evaluation criterion is same with that in the macro shooting of parallel camera configuration, people can get a better stereoscopic effect when $c$ is not exceed 2.8 and $c_{0}$ is not more than 0.91 . The above results demonstrate that $c$ and $c_{0}$ are not only suit for both stereo camera configurations, but also for any shooting distance.

\section{Comprehensive Objective Evaluation Criteria}

In the above, we have investigated the effect of individual factor on image quality for two camera configurations and various shooting distances. In the following, the overall objective evaluation criteria will be set up by combining all the individual factors.

1) Quality evaluation criteria:

The most common way to integrate all of the independent individual factor into a global index is the linear weighting method [36]-[38]. In this paper, the specified individual factors in Table VIII are independent from each other, the comprehensive objective evaluation criteria $Q$ can be gained by a linear regression equation of the quality indexes of each factor, which can be defined as

$$
Q=u \cdot Q_{p i}+v \cdot Q_{t c}+t \cdot Q_{p b}+w \cdot Q_{a}+x \cdot Q_{v}+y \cdot Q_{c}+z \cdot Q_{c 0}
$$

where $u, v, t, w, x, y$, and $z$ are the weight values of the five regions in the whole quality, restricted by $u+v+t+$ $w+x+y+z=1$, and all the weight values do not all exist simultaneously.

2) Parameter determination:

To illustrate the process of determination, we take parallel camera configuration macro shooting as an example, Pearson linear correlation coefficient (PLCC) [39] (given by Eq. (9)) is employed to evaluate prediction accuracy. Higher PLCC value indicates good correlation with human subjective judgments.

$$
P L C C(M O S, Q)=\frac{\sum_{i=1}^{n}\left(M O S_{i}-M \bar{O} S_{i}\right)\left(Q_{i}-\bar{Q}_{i}\right)}{\sum_{i=1}^{n}\left(M O S_{i}-M \bar{O} S_{i}\right)^{2}\left(Q_{i}-\bar{Q}_{i}\right)^{2}}
$$

where $n$ is the number of distorted images, $M O S_{i}$ is the subjective score of the $i$-th image and $Q_{i}$ is the objective score of the $i$-th image, $M \bar{O} S_{i}$ and $\bar{Q}_{i}$ are the mean value of subjective and objective score, respectively.

And for the nonlinear regression, we use the following fiveparameter logistic function [40]:

$$
M O S=\beta_{1} \cdot\left[\frac{1}{2}-\frac{1}{1+\exp \left(\beta_{2} \cdot\left(\chi-\beta_{3}\right)\right)}\right]+\beta_{4} \cdot \chi+\beta_{5}
$$

where $\beta_{1}, \beta_{2}, \beta_{3}, \beta_{4}$, and $\beta_{5}$ are determined by using the subjective scores and objective scores, $\chi$ is the subjective 
TABLE VIII

MEANING OF INDIVIDUAL FACTORS IN THE QUALITY EVALUATION CRITERIA

\begin{tabular}{c|l}
\hline Factor & Meaning \\
\hline$Q_{p i}$ & Output value of inter-camera distance of macro shooting factor for parallel camera configuration \\
\hline$Q_{t c}$ & Output value of convergence angle theory of macro shooting factor for toed-in camera configuration \\
\hline$Q_{p b}$ & Output value of binocular overlap percentage factor \\
\hline$Q_{a}$ & Output value of angular disparity factor in short distance shooting \\
\hline$Q_{v}$ & Output value of visual acuity of camera factor in long distance shooting \\
\hline$Q_{c}$ & Output value of ratio of foreground parallax to image width factor \\
\hline$Q_{c 0}$ & Output value of ratio of total parallax to image width factor \\
\hline
\end{tabular}

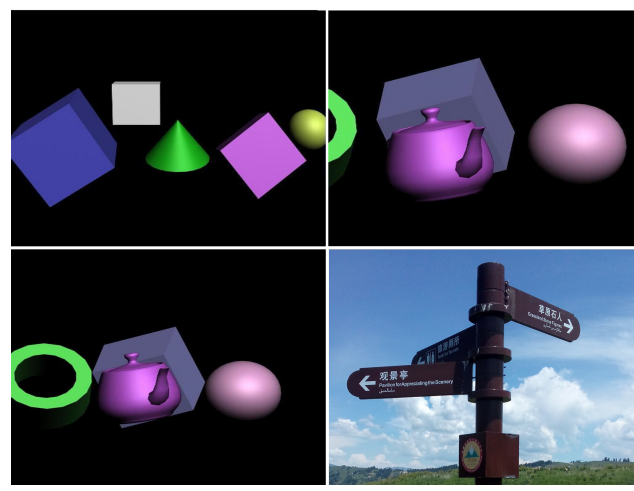

Fig. 12. Left images of four stereo images captured by parallel stereo camera macro shooting

TABLE IX

WEIGHT VALUES OF INDIVIDUAL FACTORS IN OBJECTIVE EVALUATION CRITERIA

\begin{tabular}{c|ccccccc}
\hline Type & $u$ & $v$ & $t$ & $i$ & $x$ & $y$ & $z$ \\
\hline $\begin{array}{c}\text { Macro } \\
\text { shooting for } \\
\text { parallel camera }\end{array}$ & 0.6 & 0 & 0 & 0 & 0 & 0.12 & 0.28 \\
\hline $\begin{array}{c}\text { Macro } \\
\text { shooting for } \\
\text { toed-in camera }\end{array}$ & 0 & 0.6 & 0 & 0 & 0 & 0.12 & 0.28 \\
\hline $\begin{array}{c}\text { Short distance } \\
\text { shooting for } \\
\text { parallel camera }\end{array}$ & 0 & 0 & 0.2 & 0.35 & 0 & 0.2 & 0.25 \\
\hline $\begin{array}{c}\text { Short distance } \\
\text { shooting for } \\
\text { toed-in camera }\end{array}$ & 0 & 0 & 0 & 0.4 & 0 & 0.3 & 0.3 \\
\hline $\begin{array}{c}\text { Long distance } \\
\text { shooting for } \\
\text { parallel camera }\end{array}$ & 0 & 0 & 0.2 & 0 & 0.35 & 0.2 & 0.25 \\
\hline $\begin{array}{c}\text { Long distance } \\
\text { shooting for } \\
\text { toed-in camera }\end{array}$ & 0 & 0 & 0 & 0 & 0.4 & 0.3 & 0.3 \\
\hline
\end{tabular}

evaluation score of each participant. We select a small set of the stereoscopic images database (four of the left images are shown in Fig. 12) to train the weights $u, v, y$, and $z$ in Eq. 8 to determine their values by optimizing the PLCC values between the objective and subjective scores. The results are $u=v=0.6, y=0.12$, and $z=0.28$. As mentioned above, we can obtain the rest of weight values which are shown in Table IX.

\section{3) Analysis of the proposed criteria:}

In order to examine the effect of the above used linear regression and goodness of the fit [41], we adopt three indexes: Sum of Squares Due to Error (SSE) [42], R-Square $\left(\mathrm{R}^{\wedge} 2\right)$
TABLE X

VAlues of SSE, $\mathrm{R}^{\wedge} 2$ AND RMSE of EACH ShOOTING CONDITION

\begin{tabular}{c|c|c|c|c}
\hline Stereo camera & Shooting condition & $\mathrm{SSE}$ & $\mathrm{R}^{\wedge} 2$ & $\mathrm{RMSE}$ \\
\hline \multirow{2}{*}{$\begin{array}{c}\text { Parallel } \\
\text { camera }\end{array}$} & Macro distance & 1.198 & 0.9277 & 0.33 \\
\cline { 2 - 5 } configuration & Short distance & 0.9939 & 0.9184 & 0.2041 \\
\cline { 2 - 5 } & Far distance & 0.1387 & 0.9162 & 0.2151 \\
\hline \multirow{2}{*}{$\begin{array}{c}\text { Toed-in } \\
\text { camera } \\
\text { configuration }\end{array}$} & Macro distance & 0.9248 & 0.9207 & 0.2899 \\
\cline { 2 - 5 } & Short distance & 0.6615 & 0.9554 & 0.2452 \\
\cline { 2 - 5 } & Far distance & 0.1839 & 0.9186 & 0.3033 \\
\hline
\end{tabular}

[43], and Root Mean Squared Error (RMSE) [44] (as shown in Eq. 11). Specifically, SSE [42] measures the total deviation of the response values from the fit to the response values, and the value closer to 0 indicates that the criteria has a smaller random error component, and that the fit will be more useful for prediction. $\mathrm{R}^{\wedge} 2$ [43] measures how successful the fit is in explaining the variation of the data, and if the fit is worse than just fitting a horizontal line then the value of $\mathrm{R}^{\wedge} 2$ is negative. RMSE [44] is known as the fit standard error and the standard error of the regression, and the value closer to 0 indicates a fit that is more useful for prediction.

$$
R M S E=\sqrt{\frac{1}{n} \sum_{i=1}^{n}\left(M O S_{i}-Q_{i}\right)^{2}}
$$

where $n$ is the number of stereo images, $M O S_{i}$ is the subjective score of the $i$-th image and $Q_{i}$ is the objective score of the $i$-th image, respectively.

The values of SSE, $\mathrm{R}^{\wedge} 2$, and RMSE of each shooting condition and the overall values of the completed dataset are listed in Table $X$. We can see that the values of $R^{\wedge} 2$ are all higher than 0.91, while the values of RMSE are less than 0.4, and the values of SSE are not big either. The results show that the linear regression method works well and each evaluation metric can contribute to evaluate the shooting quality. The smaller the value of SSE and RMSE, the better of the proposed quality evaluation criteria; the closer the value of $\mathrm{R}^{\wedge} 2$ to 1 , the better the performance of the metric.

\section{EXPERIMENTAL RESULTS}

In Section IV, numbers of subjective experiments are used to study the mapping between the individual influencing factors and MOS value, thus we establish the objective evaluation criteria for stereo camera according to these experimental results. To verify the proposed overall objective camera evaluation criteria in Eq. 8, another thirty non-professional adults, age from 20 to 40 , participate in the subjective assessment 


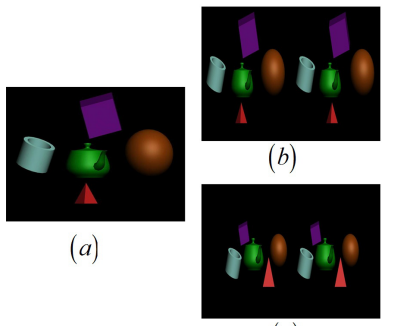

(e)

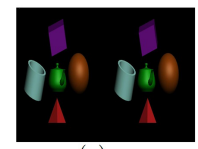

(c)

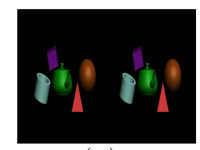

$(f)$

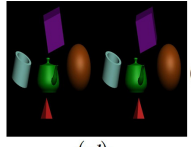

(d)

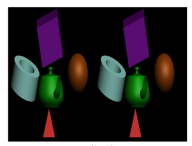

$(g)$

Fig. 13. Stereo image pairs captured by toed-in and parallel camera configurations macro shooting: (a) the selected scene; $(b)$ macro shooting with toed-in camera configuration, $d=40 \mathrm{~mm}, h=600 \mathrm{~mm}$; $(c)$ macro shooting with toed-in camera configuration, $d=40 \mathrm{~mm}, h=900 \mathrm{~mm}$; (d) macro shooting with toed-in camera configuration, $d=60 \mathrm{~mm}, h=600 \mathrm{~mm} ;(e)$ macro shooting with parallel camera configuration, $d=60 \mathrm{~mm}, h=600 \mathrm{~mm} ;(f)$ macro shooting with parallel camera configuration, $d=12 \mathrm{~mm}, h=600 \mathrm{~mm}$; $(\mathrm{g})$ macro shooting with parallel camera configuration, $d=12 \mathrm{~mm}, h=950 \mathrm{~mm}$.

experiment. All of them take the binocular visual color test and stereo vision test before the subjective experiments. They are asked to watch the same training images as mentioned in Section III.B.1).d) and then evaluate the stereo images with different camera parameters. Two hundred and forty test stereo image pairs are used in the subjective experiments and they are displayed in random order.

One of the selected scenes for stereo camera macro shooting is shown in Fig. $13(a)$. In the macro shooting of this scene, the stereo image pairs are captured by changing the value of main camera parameters $d$ and $h$, with the use of the parallel and toed-in camera configurations (Fig. $13(b)-(d)$ are the macro shooting of toed-in camera configuration, Fig. $13(e)-(\mathrm{g})$ are the macro shooting of parallel camera configuration).

Take the stereo images captured by the toed-in camera configuration as an example, shown in Fig. $13((b)-(d))$. When $d=40 \mathrm{~mm}, h=600 \mathrm{~mm}, Q$ is 4.4 . Based on the subjective experiments, the value of MOS [34] is 4.375. As can be seen, the outcome of proposed criteria is congruent with the subjective experiment result. When $d=40 \mathrm{~mm}, h=900 \mathrm{~mm}$, $Q$ is 3.9. Based on fundamental theories of our evaluation criteria, the lager the value $h$, the smaller the value of the ratio of stereo parallax to image width. The subjective experimental results present that MOS is 3.875. It reveals that our proposed criteria is consistent with the human perception. When $d=80$ $\mathrm{mm}, h=600 \mathrm{~mm}, Q$ is 2.34 . Based on the convergence angle theory, the output value is proportional to the value of $h$, and inversely proportional to the value of $d$, thus $Q$ will increase. The result of the subjective experiments shows that MOS is 2.26 and is close to the value obtained from the objective evaluation criteria.

Compared to the toed-in camera configuration shooting situation, stereo images captured by parallel camera configuration are shown in Fig. $13((e)-(\mathrm{g}))$. When $d=60 \mathrm{~mm}, h=600 \mathrm{~mm}$, $Q$ is 1.0 and MOS is 1.50 , which is in line with the output of our proposed criteria. When $d=12 \mathrm{~mm}, h=600 \mathrm{~mm}, Q$ is 4.6, and MOS is 3.83. The results reveal that the value of $d$ has a great effect on the image quality of macro shooting, which is consistent with our proposed criteria. When $d=12$

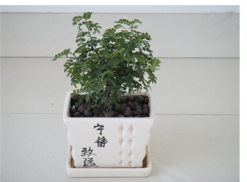

(a)

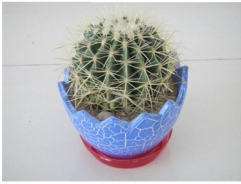

$(d)$

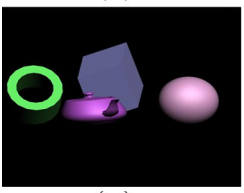

$(g)$

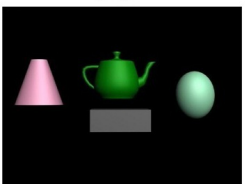

$(j)$

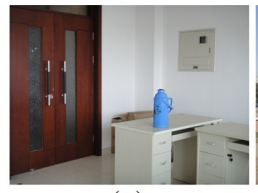

(b)

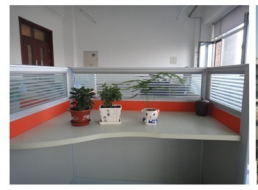

$(e)$

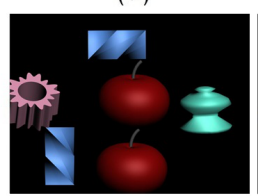

$(h)$

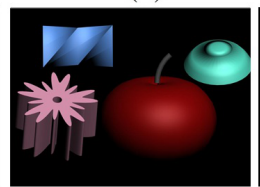

(k)

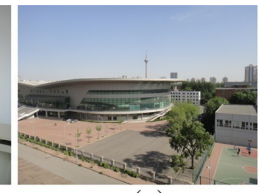

(c)

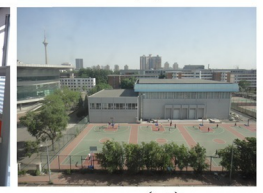

$(f)$

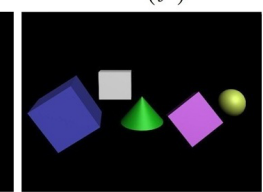

(i)

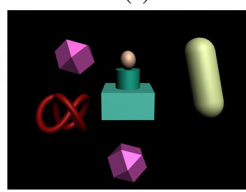

(l)
Fig. 14. Left view of stereo image pairs by real camera and 3ds Max: images $(a)-(f)$ are for real camera shooting; images $(\mathrm{g})-(l)$ are for $3 \mathrm{ds}$ Max shooting; $(a)$, (g) macro shooting with toed-in camera configuration; (b), (h) short distance shooting with toed-in camera configuration; $(c),(i)$ long distance shooting with toed-in camera configuration; $(d),(j)$ macro shooting with parallel camera configuration; $(e),(k)$ short distance shooting with parallel camera configuration; $(f),(l)$ long distance shooting with parallel camera configuration.

TABLE XI

Comparison Between SubJective and Objective Evaluation

\begin{tabular}{c|ccc}
\hline Index & Parameters in scene & $Q$ & MOS \\
\hline Short distance & $d=65 \mathrm{~mm}, h=4 \mathrm{~m}$ & 3.28 & 3.0 \\
shooting with & $d=65 \mathrm{~mm}, h=7 \mathrm{~m}$ & 4.6 & 3.73 \\
parallel camera & $d=100 \mathrm{~mm}, h=4 \mathrm{~m}$ & 1.0 & 1.55 \\
\hline Short distance & $d=65 \mathrm{~mm}, h=2.5 \mathrm{~m}$ & 4.8 & 4.375 \\
shooting with & $d=100 \mathrm{~mm}, h=2.5 \mathrm{~m}$ & 4.4 & 4.25 \\
toed-in camera & $d=65 \mathrm{~mm}, h=5 \mathrm{~m}$ & 5 & 4.5 \\
\hline Long distance & $d=420 \mathrm{~mm}, h=20 \mathrm{~m}$ & 5.0 & 4.8 \\
shooting with & $d=420 \mathrm{~mm}, h=10 \mathrm{~m}$ & 3.4 & 3.17 \\
parallel camera & $d=6 \mathrm{~m}, h=100 \mathrm{~m}$ & 2.44 & 2.5 \\
\hline Long distance & $d=9 \mathrm{~m}, h=50 \mathrm{~m}$ & 1.0 & 1.0 \\
shooting with & $d=9 \mathrm{~m}, h=100 \mathrm{~m}$ & 4.4 & 4.75 \\
toed-in camera & $d=3 \mathrm{~m}, h=5 \mathrm{~m}$ & 4.3 & 4.375 \\
\hline
\end{tabular}

$\mathrm{mm}, h=950 \mathrm{~mm}, Q$ is 5.0 . This is because with the increase of foreground and background parallax which is caused by the decrease of $d / h$ value and the increase of $h$ value, the stereo effect of images is further improved. The subjective experimental results show that MOS is 4.6 , and close to the value obtained from the objective criteria.

The subjective and objective evaluation results in short and long distance shooting are summarized in Table XI, which demonstrate the feasibility of the proposed criteria in this paper.

For ulteriorly verification of the feasibility and validity of the proposed objective criteria in this paper, another twelve groups of experimental scenes are chosen, including the six 


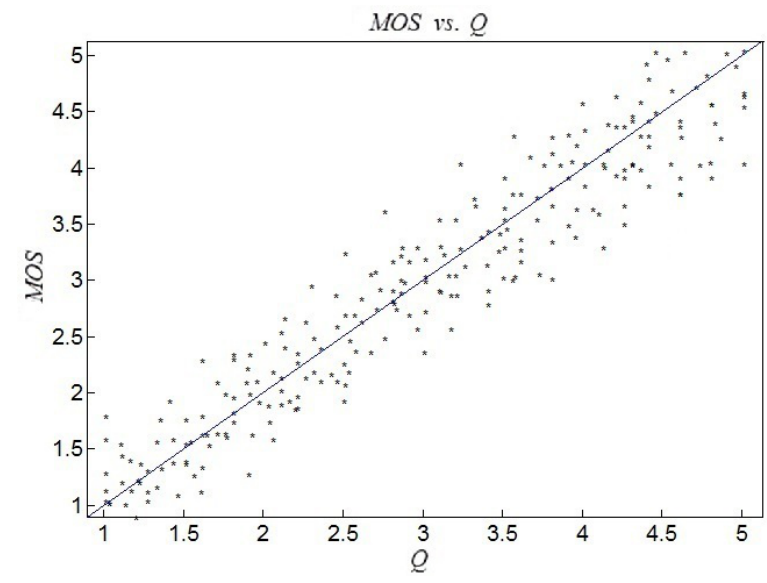

Fig. 15. Correlation between objective and subjective evaluation values schematic diagram

groups of 3D scene pictures (shown in Fig. $14(a)-(f)$ ) of real camera shooting, and the other six groups of 3D scene pictures (shown in Fig. $14(\mathrm{~g})-(l)$ ) of Autodesk 3ds Max. By changing the values of shooting parameters $h, d, m, n, l$ and $L_{\min }$ of twelve group scenes in the experiment, image pairs are captured for the validity test of the proposed criteria. The linear correlation between the objective evaluation result $Q$ and subjective evaluation result MOS values are shown in Fig. 15. The consistency between the proposed criteria and the subjective evaluation is clearly identified in the figure.

Another four commonly used performance indicators are also employed to further evaluate the proposed metric as suggested by VQEG [39]: Pearson Linear Correlation Coefficients (PLCC), Spearman Rank Order Correlation Coefficient (SROCC), Kendall Rank-Order Correlation Coefficient (KROCC), and RMSE. Among these four indicators, PLCC and RMSE, shown in Eq. 9 and Eq. 11 reflect the predicted accuracy of objective evaluation criteria, and SROCC and KROCC, shown in Eq. 12 and Eq. 13, are used to assess prediction monotonicity [45], [46]. For a perfect match between the objective and subjective scores, the following should keep valid: $\mathrm{PLCC}=\mathrm{SROCC}=\mathrm{KROCC}=1$ and $\mathrm{RMSE}=0$ [47]. Table XII presents the overall performance of the proposed quality evaluation criteria.

$$
S R O C C=1-\frac{6 \sum_{i=1}^{n} d_{i}^{2}}{n(n-1)}
$$

where $n$ is the number of stereo images, $d_{i}$ is the different between the $i$-th image's ranks in the subjective and objective evaluation. KROCC is defined as:

$$
K R O C C=\frac{n_{c}-n_{d}}{0.5 n(n-1)}
$$

where $n_{c}$ is the number of concordant pairs in the data set and $n_{d}$ is the number of discordant pairs in the data set.

What's more, we adopt two index, $\mathrm{R}^{\wedge} 2$ [43] and Fleiss' kappa [48], to present the correlation between subjective values and objective values, and measure how successful the fit is in explaining the variation of the data. Here Fleiss' kappa is a statistical measure for assessing the reliability of agreement
TABLE XII

Overall Performance of the Proposed Quality Evaluation CRITERIA

\begin{tabular}{c|c|c|c|c}
\hline Index & PLCC & SROCC & KROCC & RMSE \\
\hline Complete dataset & 0.9493 & 0.9504 & 0.8036 & 0.3695 \\
\hline
\end{tabular}

between a fixed number of participants. Conventionally, a Kappa of $<0.2$ is considered poor agreement, 0.21-0.4 fair, 0.41-0.6 moderate, 0.61-0.8 strong, and more than 0.8 near complete agreement [49]. In our paper, $\mathrm{R}^{\wedge} 2$ equals to 0.9012 which indicates a good quality, and Fleiss' kappa is 0.79 which indicates the strong agreement.

Although we could not compare our work with others as no previous studies have been conducted on shooting quality, however, from the above analysis, we can draw a conclusion that the objective evaluation results of the proposed criteria in this paper are consistent with those of subjective evaluation. With the combination of subjective experiments and theoretical analysis, the proposed criteria are applicable to evaluate the shooting quality of stereo cameras.

\section{CONCLUSION}

In this paper, we propose an objective evaluation criteria that can assess the shooting quality of stereo cameras by investigating the relationship between shooting quality and stereo camera parameters. We fully analyze the effect of individual influencing factors on shooting quality by comparing with the results obtained from extensive subjective quality tests. The experimental results show the effectiveness of the proposed quality metric in matching with the subjective ratings. There are two important contributions in this paper. First, the proposed method can be used to guide the 3D photography to capture good quality stereoscopic images. Second, the proposed criteria can also be taken as a rational setting principle of shooting parameters for the amateur. Because the previous studies only take one or a limited number of individual factors into consideration to study their effects on shooting quality, therefore they do not establish the evaluation criteria for stereo camera shooting quality which we can make a comparison with. Further studies will focus on the effect of other parameters, e.g. viewing condition and display size, to establish the comprehensive evaluation criteria with all the shooting parameters.

\section{REFERENCES}

[1] G.T. Michailidis, R. Pajarola, and I. Andreadis, "High Herformance Stereo System for Dense 3-D Reconstruction," IEEE Transactions on Circuits and Systems for Video Technology, vol. 24, no. 6, pp. 929-941, Jun. 2014.

[2] M. Stommel, M. Beetz, and W.L. Xu, "Inpainting of Missing Values in the Kinect Sensor's Depth Maps Based on Background Estimates", IEEE Sensors Journal, vol. 14, no. 4, pp.1107-1116, Apr. 2014.

[3] F. Fooladgar, S. Samavi, and S. Soroushmehr, et al., "Geometrical Analysis of Localization Error in Stereo Vision Systems", IEEE Sensors Journal, vol. 13, no. 11, pp. 4236-4246, Nov. 2013.

[4] Y. Zhang, X. Ji, and H. Wang, et al., "Stereo Interleaving Video Coding with Content Adaptive Image Subsampling," IEEE Transactions on Circuits and Systems for Video Technology, vol. 23, no. 7, pp. 1097-1108, Jul. 2013 
[5] C.T.E.R. Hewage and M.G. Martini, "Reduced-Reference Quality Assessment for 3D Video Compression and Transmission," IEEE Transactions on Consumer Electronics, vol. 57, no. 3, pp. 1185-1193, Aug. 2011.

[6] H. Liu and I. Heynderickx, "Visual Attention in Objective Image Quality Assessment: based on Eye-Tracking Data," IEEE Transactions on Circuits and Systems for Video Technology, vol. 21, no. 7, pp. 971-982, Jul. 2011.

[7] E.Y. Chen and S. Lu, "Brief Introduction of Three-Dimensional Imaging Shooting and Reproduction Principle," Television Engineering, vol. 3, pp. 25-30, 2010.

[8] S.M. Wang, "The Key Technology of Stereoscopic Photography," Motion Picture and Video Technology, vol. 2, pp. 8-11, 1998.

[9] D. Xu, L. Coria, and P. Nasiopoulos, "Guidelines for Capturing High Quality Stereoscopic Content based on a Systematic Subjective Evaluation," Electronics Circuits and Systems (ICECS), 2010 17th IEEE International Conference on, pp. 162-165, Athens, Dec. 2010.

[10] H. Yamanoue, M. Okui, and I. Yuyama, "A Study on the Relationship between Shooting Conditions and Cardboard Effect of Stereoscopic Images," IEEE Transactions on Circuits and Systems for Video Technology, vol. 10, no. 3, pp. 411-416, Apr. 2000.

[11] H. Hubert, B. Stabernack, and F. Zilly, "Architecture of a Low Latency Image Rectification Engine for Stereoscopic 3-D HDTV Processing," IEEE Transactions on Circuits and Systems for Video Technology, vol. 23, no. 5, pp. 813-822, May 2013.

[12] Z.J. Yu and S.F. Lu, "The Research on Modeling of Stereo Vision Measurement and System Accuracy in Non-ideal Situation," Applied Mechanics and Materials, vol. 121, pp. 4837-4841, Oct. 2011.

[13] M. Hasmanda and K. Riha, "The Modelling of Stereoscopic 3D Scene Acquisition," Radio Engineering, vol. 21, no. 1, pp. 134-142, Apr. 2012.

[14] W.A. IJsselsteijn, H. de Ridder, and J. Vliegen, "Subjective Evaluation of Stereoscopic Images: Effects of Camera Parameters and Display Duration," IEEE Transactions on Circuits and Systems for Video Technology, vol. 10, no. 2, pp. 225-233, Mar. 2000.

[15] H. Yamanoue, M. Okui, and F. Okano, "Geometrical Analysis of PuppetTheater and Cardboard Effects in Stereoscopic HDTV Images," IEEE Transactions on Circuits and Systems for Video Technology, vol. 16, no. 6, pp. 744-752, Jun. 2006.

[16] D. Kim and K. Sohn, "Visual Fatigue Prediction for Stereoscopic Image," IEEE Transactions on Circuits and Systems for Video Technology, vol. 21, no. 2, pp. 231-236, 2011.

[17] S. Kishi, N. Abe, and T. Shibata, et al., "Stereoscopic Camera System with Creator-Friendly Functions," SPIE Electronic Imaging, International Society for Optics and Photonics, pp. 72371M-72371M, San Jose, Jan. 2009.

[18] J. Lee, K. Chae, and S. Ji, "A Research on Controlling ThreeDimensional Effect of the Video Frames Obtained from Stereo Camera," IEEE 8th International Conference on Information, Communications and Signal Processing (ICICS), vol. 13, no. 16, pp. 1-5, Singapore, Dec. 2011.

[19] D.M. Hoffman, A.R. Girshick, and K. Akeley, et al., "VergenceAccommodation Conflicts Hinder Visual Performance and Cause Visual Fatigue," Journal of Vision, vol. 8, no. 3, pp. 1-30, Mar. 2008.

[20] J. Wattie, Bercovitz Formulae for stereo base. (2012) [Online]. Available: http://nzphoto.tripod.com/stereo/3dtake/fbercowitz.htm

[21] W. Chen, J. Fournier, and M. Barkowsky, et al., "New Stereoscopic Video Shooting Rule based on Stereoscopic Distortion Parameters and Comfortable Viewing Zone," SPIE Electronic Imaging, International Society for Optics and Photonics, pp. 786310-786310-13, California, Feb. 2011.

[22] B. Mendiburu, "3D Movie Making: Stereoscopic Digital Cinema from Scrip to Screen," CRC Press, 2009.

[23] H. Yamanoue, "The Differences between Toed-in Camera Configurations and Parallel Camera Configurations in Shooting Stereoscopic Images," Multimedia and Expo, 2006 IEEE International Conference on, pp. 111704, Toronto, Jul. 2006

[24] G.R. Jones, D. Lee, and N.S. Holliman, et al., "Controlling Perceived Depth in Stereoscopic Images," Photonics West 2001-Electronic Imaging, International Society for Optics and Photonics, pp. 42-53, San Jose, Jan. 2001.

[25] J.S. Chiang, C.H. Hsia, and H.W. Hsu, "A Stereo Vision-Based SelfLocalization System”, IEEE Sensors Journal, vol. 13, no. 5, pp. 1677 1689, May 2013.

[26] D. Scaramuzza, A. Harati, and R. Siegwart, "Extrinsic Self Calibration of a Camera and a 3D Laser Range Finder from Natural Scenes," Intelligent Robots and Systems, IROS 2007, IEEE/RSJ International Conference on, pp. 4164-4169, San Diego, Oct.-Nov. 2007.

[27] G. Lao and P. Zhu, "The Stereoscopic Photos Depth of Field Formulas and Three-Dimensional Photographic Depth of Field Positioning Feet," State Property Office of the People's Republic of China, 2003.
[28] D. Xu, L. Coria, and P. Nasiopoulos, "Quality of Experience for the Horizontal Pixel Parallax Adjustment of Stereoscopic 3D Videos," Consumer Electronics (ICCE), 2012 IEEE International Conference on, pp. 394-395, Las Vegas, Jan. 2012.

[29] ITU-R, "Subjective Assessment of Stereoscopic Television Pictures," document ITU Rec.BT.1438, 2000.

[30] Bt-500-11: Methodology for the Subjective Assessment of the Quality of Television Pictures, International Telecommunication Union, 2002.

[31] J.S. Lee, L. Goldmann, T. Ebrahimi, "Paired Comparison-Based Subjective Quality Assessment of Stereoscopic Images," Multimedia Tools and Applications, vol. 67, no. 1, pp. 31-48, 2013.

[32] A.K. Moorthy, C.C. Su, A.Mittal, et al., "Subjective Evaluation of Stereoscopic Image Quality," Signal Processing- Image Communication, vol. 28 , no. 8, pp. 870-883, 2013.

[33] T. Kawano, et al., "Performance Comparison of Subjective Assessment Methods for Stereoscopic 3D Video Quality," IEICE transactions on communications, vol. 97, no. 4, pp. 738-745, 2014.

[34] J. Seo, X. Liu, and D. Kim, et al., "An Objective Video Quality Metric for Compressed Stereoscopic Video," Circuits Systems and Signal Processing, vol. 31, no. 3, pp. 1089-1107, Jun. 2012.

[35] S. Boslaugh and P. A. Watters, "Statistics in a Nutshell," O' Reilly Media, Inc., 2008, pp. 151-168.

[36] F. Shao, W. Lin, and S. Gu, et al., "Perceptual Full-Reference Quality Assessment of Stereoscopic Images by Considering Binocular Visual Characteristics," IEEE Transactions on Image Processing, vol. 22, no. 5, pp. 1940-1953, May 2013.

[37] T. Brandão, and M.P. Queluz, "No-Reference Quality Assessment of H.264/AVC Encoded Video," IEEE Transactions on Circuits and Systems for Video Technology, vol. 20, no. 11, pp. 1437-14, Nov. 2010.

[38] X. Wan and G. Xu, "Camera Parameters Estimation and Evaluation in Active Vision System," Pattern Recognition, vol. 29, no. 3, pp. 439-447, Mar. 1996.

[39] VQEG, Final Report from the Video Quality Experts Group on the Validation of Objective Models of Video Quality Assessment. (2000) [Online]. Available: http://www.vqeg.org.

[40] P.G. Gottschalk and J.R. Dunn, "The Five-Parameter Logistic: A Characterization and Comparison with the Four-Parameter Logistic," Anal. Biochem, vol. 343, no. 1, pp. 54-65, Aug. 2005.

[41] R.L. Buchanan, R.C. Whiting, and W.C. Damert, "When is Simple Good Enough: A Comparison of the Gomperts, Baranyi, and Threephases Models for Fitting Bacterial Curves," Food Microbiology, vol. 14, no. 4, pp. 313-326, Aug. 1997.

[42] N.R. Draper and H. Smith, "Applied Regression Analysis, 3rd Edition," New York, 736pages, Apr. 1998

[43] A.M. Razali and A.A. Al-Wakeel, "Mixture Weibull Distributions for Fitting Failure Times Data," Applied Mathematics and Computation, vol. 219, no. 24, pp. 11358-11364, Aug. 2013.

[44] J.A. Carta and P. Ramírez, "Analysis of Two-Component Mixture Weibull Statistics for Estimation of Wind Speed Distributions," Renewable Energy, vol. 32, no. 3, pp. 518-531, Mar. 2007.

[45] S. Ryu and K. Sohn, "No-Reference Quality Assessment for Stereoscopic Images based on Binocular Quality Perception," IEEE Transactions on Circuits and Systems for Video Technology, vol. 24, no. 4, pp. 591-602, Apr. 2014.

[46] Y.H. Lin and J.L. Wu, "Quality Assessment of Stereoscopic 3D Image Compression by Binocular Integration Behaviors," IEEE Transaction on Image Processing, vol. 23, no. 4, pp. 1527-1541, Apr. 2014.

[47] Z.M.P. Sazzad, R. Akhter, and J. Baltes, et al., "Objective No-Reference Stereoscopic Image Quality Prediction based on 2D Image Features and Relative Disparity," Advances in Multimedia, vol. 2012, pp. 1-16, Mar. 2012.

[48] J.L. Fleiss and J. Cohen, "The Equivalence of Weighted Kappa and the Intraclass Correlation Coefficient as Measures of Reliability," Educational and Psychological Measurement, vol. 33, pp. 613-619, 1973.

[49] Cohen's and Fleiss's Kappa Explained. [Online]. Available: http://www.statstodo.com/CohenKappa_Exp.php 


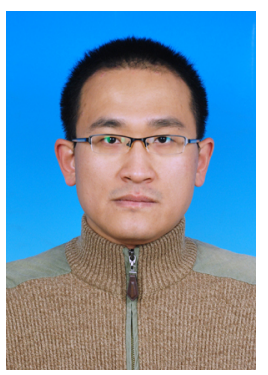

Jiachen Yang received the M.S. and Ph.D. degrees in Communication and Information Engineering from the Tianjin University, Tianjin, China, in 2005 and 2009, respectively. $\mathrm{He}$ is an associate professor at Tianjin University. Now, he is a visiting scholar in the Department of Computer Science, School of Science at Loughborough University, UK. His research interests include stereo camera, stereo vision research, pattern recognition, stereo image displaying and quality evaluation.

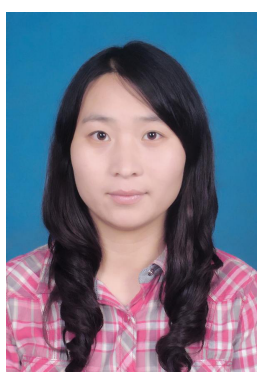

Yun Liu is a Ph.D. student with School of Electronic Information Engineering, Tianjin University, Tianjin, China. Her research interests include stereo camera, stereo vision research, stereo image displaying and quality evaluation.

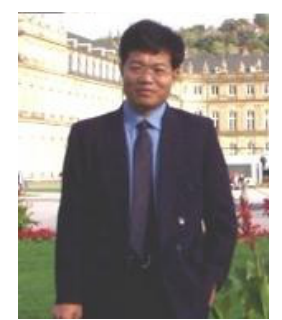

Qinggang Meng received B.S. and M.S. degrees in School of Electronic Information Engineering, Tianjin University, China, and Ph.D. degree in Computer Science from Aberystwyth University, UK. He is a Senior Lecturer in the Department of Computer Science, Loughborough University, UK. His research interests include biologically and psychologically inspired learning algorithms and developmental robotics, service robotics, robot learning and adaptation, multi-UAV cooperation, drivers distraction detection, human motion analysis and activity recognition, activity pattern detection, pattern recognition, artificial intelligence and computer vision. He is a member of the IEEE and a fellow of the Higher Education Academy of United Kingdom.

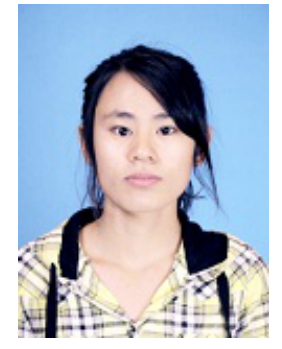

Rongrong Chu is a M.S. student with School of Electronic Information Engineering, Tianjin University, Tianjin, China. Her research interests include stereo camera and stereo vision research. 